\title{
Uma abordagem multicritério para a priorização de vias pavimentadas
}

\author{
Josiane Palma Lima'; Rui António Rodrigues Ramos²; José Leomar Fernandes Júnior ${ }^{3}$
}

\begin{abstract}
Resumo: Este trabalho apresenta um modelo de priorização de vias que integra métodos de avaliação multicritério em ambiente de Sistema de Informação Geográfica (SIG). Critérios objetivos e subjetivos são utilizados como base no apoio à tomada de decisão. O estudo de caso desenvolvido para a cidade de São Carlos - SP possibilitou a exploração do modelo com o desenvolvimento de cenários de avaliação, gerados através da combinação de critérios pelos métodos de WLC (Weighted Linear Combination) e OWA (Ordered Weighted Average), com intuito de integrar os riscos na análise. Os diversos cenários foram gerados com o objetivo de priorizar as vias pavimentadas com relação à sua localização, condição do pavimento, hierarquia viária e custos das estratégias previstas. O modelo mostrou-se eficiente em várias etapas do processo, destacando-se o fato de considerar de forma simples os conceitos de priorização de vias e integrá-los numa ferramenta poderosa de análise espacial.
\end{abstract}

\begin{abstract}
This work presents urban roadways prioritization model that integrates Multicriteria Decision Analysis and Geographical Information System (GIS). Objective and subjective criteria are used as base in the support decision. The case study performed at the city of Sao Carlos, State of Sao Paulo, Brazil, made possible the exploration of the model with the development of evaluation sceneries, through the methods of Weighted Linear Combination (WLC) and Ordered Weighted Average (OWA), integrating the risks analysis. The various sceneries were development based on pavement condition, roads hierarchy, costs of strategies and location. The model was shown efficient in various stages of the process, because it considers the concepts of roads prioritization and it integrates them in a powerful tool of space analysis.
\end{abstract}

\section{INTRODUÇÃO}

A gestão das atividades de conservação de vias por um órgão responsável traz benefícios aos usuários de rodovias e vias urbanas e à população, pois os recursos públicos destinados à conservação destas vias são aplicados com maior eficiência. Também, a administração, em seus diversos níveis é beneficiada devido à maior possibilidade de se adotar decisões corretas e coordenadas entre os diversos setores do órgão responsável, público ou privado. Entretanto, Visconti (2000) comenta que os principais fatores externos condicionantes de um sistema de gestão de vias urbanas são os recursos orçamentários, os dados necessários ao sistema e as diretrizes políticas e administrativas. As vias pavimentadas representam um valioso patrimônio cuja conservação e restauração são essenciais para a sua preservação.

A definição das prioridades de conservação deve ser realizada considerando todos os fatores técnicos e econômicos, não apenas a curto prazo, mas também analisando as conseqüências de todos os custos e bene-

\footnotetext{
$\overline{1}$ Josiane Palma Lima, Universidade Federal de Itajubá, Instituto de Engenharia de Produção e Gestão, Itajubá , MG, Brasil. (e-mail: jplima@unifei.edu.br).

${ }^{2}$ Rui António Rodrigues Ramos, Universidade do Minho, Departamento de Engenharia Civil, Minho, Portugal. (e-mail: rramos@civil.uminho.pt).

${ }^{3}$ José Leomar Fernandes Júnior, Universidade de São Paulo, Escola de Engenharia de São Carlos, São Carlos, SP, Brasil. (e-mail: leomar@sc.usp.br).
}

Manuscrito recebido em 2/7/2008 e aprovado para publicação em 29/6/2009. Este artigo é parte de TRANSPORTES, volume XVII, número 1 , junho de 2009. ISSN: 1415-7713. fícios a médio prazo. Isso se faz através da utilização de sistemas de gerência que permitam, através dos respectivos modelos de desempenho, simular os efeitos de diferentes estratégias de conservação. No entanto, mesmo na ausência destes meios de apoio à simulação de diferentes cenários (os modelos de desempenho), existe a possibilidade de determinar as prioridades de conservação através de um conjunto de critérios de compreensão bastante racional (Pereira \& Miranda, 1999).

Atualmente, há vários modelos propostos, utilizando diferentes critérios, para a definição das prioridades de manutenção e reabilitação (M\&R) de uma rede de vias pavimentadas. Quase sempre os órgãos responsáveis utilizam modelos de priorização que consideram as intervenções anuais, através do ranking de projetos candidatos às atividades de $M \& R$ em cada ano do período de análise. Estes métodos desenvolvem índices de prioridades utilizando formulações matemáticas, baseadas em um fator ou uma combinação de fatores de priorização. A partir de uma lista de projetos prioritários os engenheiros de pavimentos podem justificar os gastos nos investimentos em manutenção de pavimentos. Encontram-se vários trabalhos recentes (Chen et al., 1993; Misra et al., 2003, Mohammad et al., 2004; Chandran et al., 2007; Albuquerque, 2007) utilizando índices de prioridades de intervenção, indicando que o tema ainda continua motivando o interesse para o desenvolvimento de novas pesquisas. $\mathrm{O}$ que se observa claramente em vários trabalhos acerca do tema é que, as seções prioritárias herdam com naturalidade um caráter que leva a medi- 
das ou avaliação envolvendo inúmeros atributos, razão pela qual se justifica o desenvolvimento de um modelo fundamentado em métodos de avaliação multicritério para obtenção dos seus índices.

O presente trabalho aborda à questão da priorização de vias pavimentadas utilizando-se de componentes espaciais de análise (com o uso de Sistema de Informação Geográfica - SIG) e o estudo de critérios de decisão, muitos deles subjetivos (com as técnicas de Avaliação Multicritério, do inglês Multicriteria Decision Analysis - MCDA). Por este motivo, os SIGs se apresentam, observando a literatura especializada (Jankowski et al., 2001; Costa et al., 2004; Rodrigues et al., 2004; Zambon, 2004), como a plataforma ideal para a aplicação da técnica de avaliação multicritério. Pelo seu lado, a avaliação multicritério oferece uma vasta coleção de técnicas e procedimentos que permitem revelar as preferências dos decisores e incorporálas em tomadas de decisão baseadas em SIG. Os SIGs, por sua vez, integram técnicas de gestão de dados que permitem gerir e manusear a informação em que se baseiam os índices (Lima, 2007; Mello et al. 2001; Ramos, 2000).

Portanto, é objetivo deste trabalho apresentar um modelo de priorização de vias, desenvolvido por Lima (2007), que integra métodos de avaliação multicritério em ambiente de Sistema de Informação Geográfica (SIG). Critérios objetivos e subjetivos são utilizados como base no apoio à tomada de decisão. Através de um estudo de caso desenvolvido para a cidade de São Carlos - SP foi possível identificar as potencialidades deste modelo no apoio à decisão na gestão de vias pavimentadas.

\section{MODELO DE PRIORIZAÇÃO DE VIAS PAVIMENTADAS}

A priorização de vias pode ser desenvolvida de acordo com diferentes pressupostos, em função do desígnio que se pretende alcançar e das respectivas variáveis associadas. De modo geral, o estudo desenvolvido abrange duas visões distintas, que podem ser consideradas autonomamente ou em conjunto, na identificação dessas priorizações. A primeira trata-se de uma visão socioeconômica, em que projetos consideram soluções estratégicas, buscando minimizar o custo das intervenções e maximizar o benefício dos usuários, no sentido de melhorar a qualidade de vias de acesso a locais específicos. E, a segunda, trata-se de uma visão técnica, em que a meta é manter a rede em boas condições para circulação do tráfego, mas procurando também aumentar a vida útil dos pavimentos. $\mathrm{O}$ modelo apresenta um índice para as seções e/ou vias pavimentadas que atende a critérios relativos à importância da seção na rede, tanto pela sua localização como pela sua classificação, as condições do pavimento e aos custos associados às estratégias de M\&R aplicáveis. As técnicas de Avaliação Multicritério (MCDA- Multicriteria Decision Analysis ) são utilizadas para quantificar a importância dos vários critérios e/ou grupos de critérios, ou seja, o quanto eles influenciam a necessidade de M\&R de pavimentos. $\mathrm{O}$ processo considera múltiplos critérios organizados em vários níveis de uma estrutura hierárquica, em grupos e subgrupos de critérios em que o nível mais alto deverá ser a meta do problema, o objetivo final do processo de decisão.

A escolha do método MCDA faz parte da fase de estruturação do problema e os decisores precisam concordar plenamente com o método escolhido. Existem vários métodos MCDA, utilizados com sucesso em diversas situações, como por exemplo, o MAUT Multi-Attribute Utility Theory e o AHP - Analytic Hierarchy Process, bastante utilizados em problemas de transportes, o ELECTRE - Elimination and choice translating algorithm, o PROMETHEE - Preference Ranking Method for Enrichment Evaluation e o MABETH - Measuring Attractiveness by a Categorical Based Evaluation Technique (Saaty, 1977; Bana e Costa \& Vasnick, 1994 e 2007; Salomon et al., 1999; Zietsman et al., 2003). Os vários métodos diferem no modo como as preferências em relação aos vários atributos são especificadas e no modo como as alternativas são ordenadas (Cafiso et al., 2002).

\subsection{Identificação e avaliação dos critérios de decisão}

A tomada de decisão de âmbito espacial e multicritério requer uma articulação entre os objetivos de um ou mais decisores e a identificação dos atributos necessários na determinação do grau em que esses objetivos serão atingidos. Um atributo é medido de acordo com o seu desempenho em relação ao objetivo. Ao objetivo e aos respectivos atributos, por formarem uma estrutura hierárquica de critérios de avaliação para um determinado problema de decisão, devem ser atribuídos um peso, que virá representar a importância relativa de cada um em relação a sua contribuição na obtenção de um índice global. Portanto, primeiramente foi definido um conjunto de critérios (ou atributos) de decisão efetivamente relevantes, estabelecidos para o modelo de priorização, com base em questionários e opinião de especialistas e profissionais responsáveis por obras e serviços relacionados com os pavimentos urbanos (Lima, 2007), conforme apresentado na Tabela 1. Depois de estruturado o conjunto de critérios que fará parte do processo de avaliação é necessário definir a importância que cada critério e grupos de critérios têm em relação ao objetivo almejado, ou seja, a priorização de vias pavimentadas. 
De um conjunto de procedimentos para a definição de pesos, estabelecidos e utilizados por diversos autores, alguns dos mais usuais são (Malczewski, 1999):

- Escala de n pontos (introduzido por Osgood et al.,1957);

- Distribuição de pontos e sua variante, estimativa de rácios (Easton, 1973);

- Analytic Hierarchy Process - AHP (Saaty, 1980);

- Rank sum e rank reciprocal (Stillwell et al., 1981), baseados na ordenação de critérios.

Conforme Cafiso et al. (2002), o AHP parece ser uma das técnicas que mais se adapta nas aplicações e decisões na gerência de infra-estruturas viárias, mostrando-se satisfatório na integração com os Sistemas de Gerência de Pavimentos (SGPs), como por exemplo o HDM-4 (Highway Development and Management Tool), porque produz um índice baseado num ranking com medida do desempenho das alternativas. Finalmente, no modelo apresentado, para a definição dos pesos dos critérios adotou-se o método de comparação par a par proposto por Saaty (1980) no Processo Analítico Hierárquico (do inglês Analytic Hierarchy Process - AHP) com a escala composta por nove níveis numéricos para expressar e normalizar os julgamentos efetuados, conforme a Figura 1. Por meio dessa técnica, pesos e prioridades são derivados a partir de um conjunto de julgamentos subjetivos realizados por avaliadores ou participantes envolvidos no processo.

A comparação par a par, entre os $n$ critérios, é realizada a partir de uma matriz recíproca quadrada $n \times n$, onde os critérios estão dispostos na mesma ordem ao longo das linhas e das colunas. Portanto, o valor $a_{i j}$ representa a importância do critério da linha $i$ em relação ao critério da coluna $j$, conforme a Equação 1. Para mais detalhes sobre a aplicação do método de comparação par a par de critérios, ver Ramos (2000), Lima (2007).

$$
a_{i j}= \begin{cases}\frac{1}{a_{j i}}, & \text { para } i \neq j \\ 1, & \text { para } i=j\end{cases}
$$

A Tabela 1 apresenta o conjunto de critérios agregados ao modelo. Alguns critérios não puderam ser considerados na aplicação desenvolvida devido à falta de dados confiáveis. Os pesos foram remodelados com base nos graus de importância atribuídos pelos avaliadores especialistas para os fatores em questão, sempre levando em conta o grau de consistência da avaliação.

Do conjunto de critérios mostrados na Tabela 1, juntamente com os pesos atribuídos pelos avaliadores, os critérios que não foram considerados no estudo de caso são: Nível 3- Idade desde a última intervenção, Proximidade a Portos e Aeroportos; e Nível 2 - Ques-

\begin{tabular}{|c|c|c|c|c|c|c|c|c|}
\hline $1 / 9$ & $1 / 7$ & $1 / 5$ & $1 / 3$ & 1 & 3 & 5 & 7 & 9 \\
$\begin{array}{c}\text { Extremamente } \\
\text { menos } \\
\text { importante }\end{array}$ & $\begin{array}{c}\text { Bastante menos } \\
\text { importante }\end{array}$ & $\begin{array}{c}\text { Muito menos } \\
\text { importante }\end{array}$ & $\begin{array}{c}\text { Pouco menos } \\
\text { importante }\end{array}$ & $\begin{array}{c}\text { Igual } \\
\text { importância }\end{array}$ & $\begin{array}{c}\text { Pouco mais } \\
\text { importante }\end{array}$ & $\begin{array}{c}\text { Muito } \\
\text { importante }\end{array}$ & $\begin{array}{c}\text { Bastante mais } \\
\text { importante }\end{array}$ & $\begin{array}{c}\text { Extremamente } \\
\text { mais importante }\end{array}$ \\
\hline
\end{tabular}

Figura 1. Escala de Comparação de Critérios (Saaty, 1980)

Tabela 1. Critérios de priorização, pesos, códigos e agrupamentos

\begin{tabular}{|c|c|c|c|c|c|}
\hline Código & Fatores e Grupos de Fatores & Pesos & Código & Fatores e Grupos de Fatores & Pesos \\
\hline $\mathbf{A}$ & Fatores associados à hierarquia viária & 0,223 & \multirow{2}{*}{$\mathbf{C}$} & Fatores associados à localização das & \multirow{2}{*}{0,128} \\
\hline$A 1$ & Classe funcional & 0,290 & & seções de pavimento & \\
\hline$A 2$ & Tipo de rota & 0,210 & $C 1$ & Proximidade infra-estruturas Transportes & 0,425 \\
\hline$A 3$ & Volume de tráfego & 0,500 & $\mathrm{C} 1.1$ & Proximidade a terminal rodoviário & 0,437 \\
\hline \multirow{2}{*}{ B } & Fatores associados às questões técnicas & \multirow{2}{*}{0,378} & $\mathrm{C} 1.2$ & Proximidade a terminal ferroviário & 0,195 \\
\hline & e operacionais & & $\mathrm{C} 1.3$ & Proximidade a rodovias & 0,368 \\
\hline$B 1$ & Índice combinado de defeitos & 0,592 & $C 2$ & Proximidade a equipamentos públicos & 0,304 \\
\hline B1.1 & Trincas por Fadiga & 0,134 & $\mathrm{C} 2.1$ & Proximidade a escolas primárias e secundária & 0,100 \\
\hline B1.2 & Trincas em Blocos & 0,036 & $\mathrm{C} 2.2$ & Proximidade a universidades & 0,113 \\
\hline B1.3 & Defeito nos Bordos & 0,031 & $\mathrm{C} 2.3$ & Proximidade a postos de saúde & 0,183 \\
\hline B1.4 & Trincas Longitudinais & 0,040 & $\mathrm{C} 2.4$ & Proximidade a hospitais & 0,237 \\
\hline B1.5 & Trincas por Reflexão & 0,053 & $\mathrm{C} 2.5$ & Proximidade a centros administrativos & 0,082 \\
\hline B1.6 & Trincas Transversais & 0,044 & $\mathrm{C} 2.6$ & Proximidade a corporação de bombeiros & 0,185 \\
\hline B1.7 & Remendos & 0,049 & $\mathrm{C} 2.7$ & Proximidade a facilidades militares & 0,099 \\
\hline B1.8 & Panelas & 0,211 & $C 3$ & Preferências pessoais ou administrativas & 0,271 \\
\hline B1.9 & Deformação Permanente & 0,146 & C3.1 & Proximidade a centros comerciais & 0,334 \\
\hline B1.10 & Corrugação & 0,049 & & Proximidade a bairros com particular & \\
\hline B1.11 & Exudação & 0,027 & C3. & & 0,141 \\
\hline B1.12 & Agregados Polidos & 0,023 & C3.3 & Proximidade a áreas de lazer e esportes & 0,110 \\
\hline B1.13 & Desgaste & 0,059 & C 3.4 & Proximidade a áreas turísticas & 0,234 \\
\hline B1.14 & Desnível & 0,048 & C3.5 & Proximidade a áreas industriais & 0,181 \\
\hline B1.15 & Bombeamento & 0,050 & D & Fatores associados aos custos & 0,271 \\
\hline$B 2$ & Índice da condição do pavimento & 0,410 & $\mathbf{D}$ & 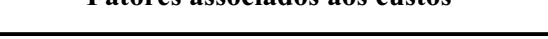 & 0,11 \\
\hline
\end{tabular}


tões Ambientais, Questões de Segurança, Custos dos Usuários. Considerar ou não algum critério do modelo proposto para uma aplicação prática não prejudica o desenvolvimento de mapas e análises dos diversos cenários de avaliação, pois se trata de um processo flexível quanto à escolha dos critérios relevantes ao propósito que se pretende atingir. Deve-se enfatizar ainda, que os critérios considerados devem ser passíveis de avaliação, tanto do ponto de vista da disponibilidade de dados quanto do ponto de vista do esforço de aquisição dos mesmos. Pode-se observar ainda na Tabela 1 que os critérios estão estruturados em quatro grandes grupos: (A) fatores associados à hierarquia viária; (B) fatores associados às questões técnicas $\mathrm{e}$ operacionais; (C) fatores associados à localização das seções de pavimentos; e (D) fatores associados aos custos.

\subsection{Desenvolvimento do índice de prioridades}

O modelo para priorização de vias urbanas pavimentadas propõe o desenvolvimento de um índice de prioridade $(I P)$ para cada seção $(i)$ da rede. O índice de prioridade é um valor que incorpora os atributos relacionados a cada seção de pavimento e resulta da combinação desses atributos, para cada critério. Este índice foi determinado através da agregação dos scores obtidos para os vários grupos $\left(\right.$ Score $\left._{g}\right)$ em avaliação. Cada Scoreg resulta da média ponderada dos scores de cada grupo (g) de fatores, que por sua vez são obtidos pela agregação dos scores dos vários fatores $\left(\right.$ Score $\left._{f}\right)$. Por sua vez, os $\mathrm{Score}_{f}$ resultam da agregação dos $\mathrm{sco}$ res normalizados dos atributos, também freqüentemente considerados como indicadores $\left(\right.$ Score $\left._{i}\right)$. No entanto, os vários critérios (fatores) em análise podem ser complementares ou equivalentes, e frente a essa situação, faz-se a agregação em grupos de critérios similares. Assim, o IP passa a ser avaliado por grupos de critérios (ou grupos de fatores), conforme a Equação 2, onde Score $_{g}$ e $w_{g}$ são respectivamente o score normalizado e ponderado e o peso atribuído a cada grupo $(g)$ de critérios.

$$
I P=\frac{\sum_{g}\left(\text { Score }_{g} \cdot w_{g}\right)}{\sum_{g} w_{g}}
$$

Portanto, para aplicar o modelo de priorização de vias pavimentadas é necessário que sejam conhecidos os seguintes dados: a rede viária existente, os critérios de decisão, os pesos a aplicar a cada critério e aos grupos de critérios, as funções de normalização e as informações pertinentes a cada critério.

\subsection{Normalização e combinação de critérios}

Os critérios envolvidos são expressos em termos de características da via ou da seção de pavimento. Essas características podem assumir valores numéricos (objetivos) ou lingüísticos (subjetivos), dependendo do tipo de critério que está sendo considerado. Para que os valores dos diferentes critérios possam ser agregados é necessário efetuar a sua normalização. Os critérios contínuos são normalizados recorrendo-se a funções fuzzy, segundo as quais um conjunto de valores expresso numa dada escala é convertido num outro, comparável e expresso numa escala normalizada (neste trabalho, de 0 a 1 ). Por outro lado, alguns critérios com valores lingüísticos subjetivos são normalizados atribuindo-se valores de scores de forma arbitrária, dentro da mesma escala adotada para os critérios contínuos. A forma arbitrária de atribuir valores de scores dentro de uma determinada escala normalizada não afeta a confiabilidade da análise, sendo que é sempre possível adaptá-la a cada aplicação, atribuindo valores e variáveis diferentes para serem estudados caso a caso. Do mesmo modo, também é possível incorporar diversas formas de funções fuzzy ao processo, sendo as mais conhecidas: sigmoidal, $\mathrm{j}$-shaped, linear e complexa (Zadeh, 1965). A função fuzzy sigmoidal, com os pontos de controle $x_{a}$ e $x_{b}$, utilizada para a normalização do critério Índice da Condição do Pavimento (Critério B2, Tabela 1), por exemplo, é apresentada na Figura 2. Uma vez normalizados os scores dos critérios, esses são combinados de acordo com a regra de decisão. Existem diversas classes de operadores para a combinação de critérios (para uma descrição extensiva ver Malczewski, 1999). Os mais utilizados nos processos de decisão espacial são a Combinação Linear Ponderada (WLC, do inglês Weighted Linear Combination) e a Média Ponderada Ordenada (OWA, do inglês Ordered Weighted Average).

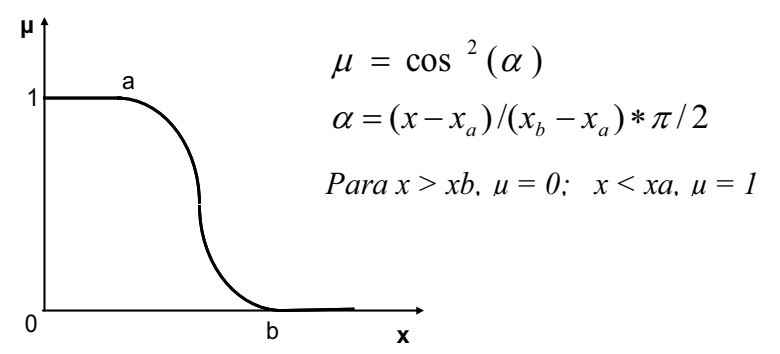

Figura 2. Exemplo de função fuzzy (sigmoidal decrescente)

A expressão que agrega todos os scores normalizados (Equação 1) corresponde a um WLC (Voogd, 1983), combinando os fatores através de uma média ponderada e permitindo aos critérios compensar entre eles as suas qualidades (Trade-off). O OWA (Yager, 1988) além de utilizar os pesos de critérios usados no procedimento WLC, considera outro conjunto de pesos. Os novos pesos denominam-se "pesos ordenados", do inglês Order Weights, uma vez que sua aplicação depende de uma determinada ordenação dos fa- 
tores que agregam (Eastman et al., 1998). Depois da aplicação do primeiro conjunto de pesos aos fatores (tal como no procedimento WLC), os scores resultantes (agora ponderados) são ordenados do valor mais baixo para o mais elevado. Ao fator com o score ponderado mais baixo (o primeiro da lista ordenada) é aplicado o primeiro order weight, ao fator com o segundo valor mais baixo é aplicado o segundo order weight, e assim sucessivamente. Trata-se, portanto, de ponderar os fatores de acordo com sua ordem, do mínimo para o máximo. Fazendo variar os order weights, o procedimento OWA permite implementar uma gama vastíssima (na verdade infinita) de operadores de agregação.

Em um processo de decisão que envolva três fatores, um conjunto order weight $\left[\begin{array}{lll}1 & 0 & 0\end{array}\right]$ aplicaria todo o peso ao fator com o menor score, produzindo assim uma solução sem risco (dita pessimista ou conservadora), equivalente ao operador lógico AND; um conjunto order weight [ $\left[\begin{array}{lll}0 & 0 & 1\end{array}\right]$, pelo contrário, aplicaria todo o peso ao fator de mais alto score, produzindo assim uma solução de elevado risco (dita otimista), equivalente ao operador lógico OR; um conjunto order weight [0,33 0,33 0,33], por sua vez, aplicaria igual peso a todos os fatores, produzindo assim uma solução de risco neutro (intermédia), equivalente ao operador WLC (Eastman et al., 1998). Nos dois primeiros casos, apenas os scores extremos são considerados (o mínimo no primeiro e o máximo no segundo), o que significa que os fatores não podem ser compensados uns pelos outros (ausência de Tradeoff). Contudo, no terceiro caso, como foi atribuído um conjunto de "pesos ordenados" perfeitamente equilibrado, os fatores podem compensar-se mutuamente (Trade-off total), no sentido em que maus scores em alguns fatores podem ser compensados por bons scores em outros. Portanto, este terceiro caso é um equivalente do WLC ou, ainda mais corretamente, o procedimento WLC é um caso particular do procedimento mais geral OWA. Qualquer combinação de order weights é possível, mas observando-se sempre a unidade do somatório. A Figura 3a representa um espectro triangular estratégico de decisão, definido pela ati- tude de risco e pelo nível de Trade-off (Ramos, 2000; Lima, 2007). A Figura $3 b$ apresenta os vários pontos de decisão utilizados neste trabalho. $\mathrm{O}$ deslocamento relativo dos order weights no sentido do mínimo ou do máximo controla o nível de risco, designado por ANDness. Por sua vez, a homogeneidade da distribuição dos order weights através das posições controla o nível global de Trade-off.

O nível de risco (ANDness) e a compensação entre scores (Trade-off) são medidos, respectivamente, através das Equações 3 e 4, onde $n$ é o número total de fatores, $i$ é a ordem de cada fator e $O_{i}$ é o peso (order weight ) do fator de ordem $i$ (Eastmann et al.,1998).

$$
\begin{aligned}
& \text { ANDness }=\frac{1}{n-1} \cdot \sum_{i}\left((n-1) \cdot O_{i}\right) \\
& \text { Tradeoff }=1-\sqrt{\frac{n \cdot \sum_{i}\left(O_{i}-\frac{1}{n}\right)^{2}}{(n-1)}}
\end{aligned}
$$

\section{PRIORIZAÇÃO DE VIAS PAVIMENTADAS - ESTUDO DE CASO REAL}

$\mathrm{O}$ modelo de priorização de vias foi aplicado à rede de vias urbanas da cidade de São Carlos (SP). O município de São Carlos está situado na região central do estado de São Paulo, Brasil, a $230 \mathrm{~km}$ da capital do estado. A cidade de São Carlos pode ser considerada uma típica cidade média brasileira e apresenta em torno de $4.000 .000 \mathrm{~m}^{2}$ de vias urbanas, organizadas ortogonalmente, sendo que aproximadamente 6.000 seções são pavimentadas $\left(2.800 .000 \mathrm{~m}^{2}\right.$ de vias com pavimento flexível).

A plataforma SIG adotada para a implementação do Estudo de Caso foi o ArcView 3.2, utilizando o formato vetorial do software, dentro do qual foi agregado o modelo desenvolvido e foram desenvolvidas todas as etapas necessárias à sua implementação. A análise espacial dos dados é uma importante aliada para esse tipo de decisão e os SIGs permitem que as informações do mundo real sejam retratadas de forma muito próxima da realidade, através da localização geográfi-

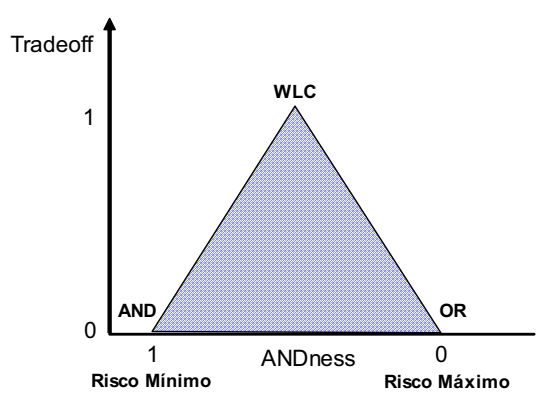

(a) Espaço Estratégico

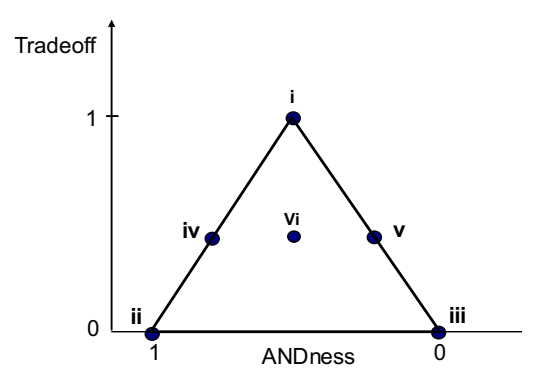

(b) Pontos de Decisão

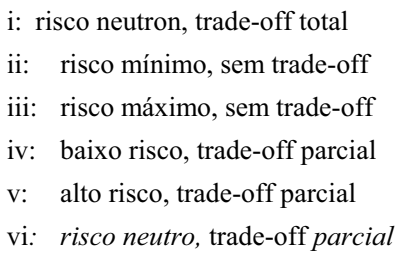

vi: risco neutro, trade-off parcial

Figura 3. Espaço estratégico e pontos de decisão - OWA 
ca. Cada seção ou segmento da rede viária é avaliada de forma independente, de acordo com seus próprios atributos.

O modelo foi desenvolvido com base numa estrutura hierárquica de decisão, que foi adaptada para o estudo de caso, conforme a Figura 4 e os códigos da Tabela 1 . O processo considera múltiplos critérios organizados em vários níveis, em grupos e subgrupos de critérios. A aplicação do modelo só é possível se todas as informações em relação aos critérios de decisão forem coletadas e tratadas para posterior utilização em SIG. Sendo assim, este trabalho contou com levantamento de campo, desenvolvimento de inventário sobre características físicas e geométricas de ruas e com a avaliação do pavimento de toda a rede da cidade (Para mais detalhes do levantamento ver Lima et al., 2004).

No intuito de gerar um conjunto de cenários de avaliação de prioridades, as combinações de fatores WLC e OWA são realizadas de acordo com cada nível e agrupamento da estrutura (Figura 4). A Tabela 2 mostra os procedimentos adotados bem como a nomenclatura dada para cada agrupamento por nível de decisão, de acordo com os códigos dos critérios da Tabela 1 e agrupamentos da Figura 4. O resultado da aplicação destes procedimentos é a produção de vários mapas contendo a rede viária da cidade de São Carlos, cada um representando um cenário que caracteriza a prioridade de intervenção para cada seção da rede. Estes cenários diferem na forma como os critérios de decisão foram combinados e cabe ao decisor escolher a melhor alternativa.

\subsection{Combinações de critérios do Grupo A (Nível 2)}

Na combinação dos critérios do Grupo A, foi realizada a combinação nomeadamente WLC_A (Tabela 2), agrupando os scores normalizados dos fatores A1, A2 e A3 do nível 2, no qual deu origem ao mapa A, representando o grupo A - Fatores Associados à Hierarquia Viária no nível 1, conforme as Figuras 5a, 5b, 5c e 5d. De acordo com a escala adotada, as seções com prioridade máxima são aquelas que possuem score máximo, ou seja, valor 1,0 e cor vermelha. De acordo com o mapa A (Figura 5d), observam-se, entre outras, que três grandes e importantes vias da cidade foram priorizadas, com score entre 0,8 e 1,0 (sendo o intervalo possível de 0 a 1,0 ), evidenciando a importância relativa da hierarquia das vias e de outras que podem ser vistas no mapa. O mapa não possui seções com scores zero (sem prioridade), com média de valores de 0,621 .

\subsection{Combinações de critérios do Grupo B (Níveis 3 e 2)}

A combinação dos critérios do Grupo B, contou primeiramente com a combinação WLC_B1 no nível 3

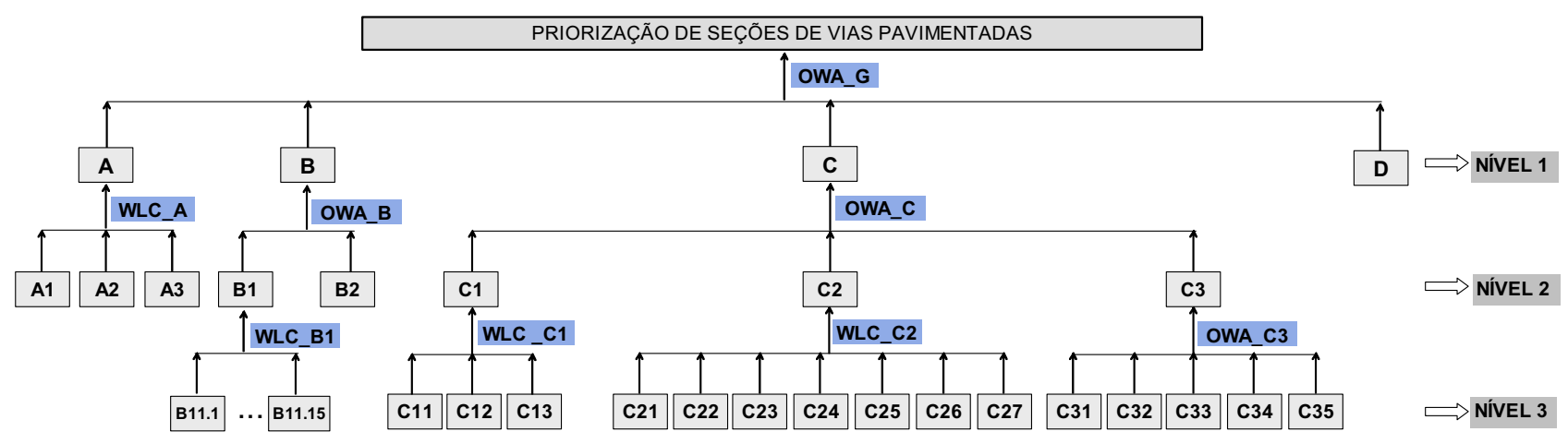

Figura 4. Estrutura hierárquica e as combinações WLC e OWA de critérios

Tabela 2. Procedimentos adotados para a combinação de critérios

\begin{tabular}{|c|c|c|c|}
\hline Nível & Combinações & Aplicação & Agrupamento \\
\hline 1 & OWA & $\begin{array}{l}\text { Combinação dos mapas do Nível 1, para todos os grupos } \\
\text { de critérios, gerando } 6 \text { cenários finais de avaliação: Pi_1, } \\
\text { Pii_1, Piii_1 e Pi_2, Pii_2 e Piii_2 }\end{array}$ & OWA_G \\
\hline \multirow{3}{*}{2} & WLC & $\begin{array}{l}\text { Combinação de mapas do nível 2, para gerar o mapa do } \\
\text { grupo A utilizando os pesos dos respectivos critérios }\end{array}$ & WLC_A \\
\hline & \multirow{2}{*}{ OWA } & \multirow{2}{*}{$\begin{array}{l}\text { Combinação dos mapas do Nível 2, gerando } 3 \text { cenários pa- } \\
\text { ra o grupo B - Bi, Bii e Biii e } 6 \text { cenários para o grupo C: } \\
\text { Ci.1, Cii.1, Ciii.1 e Ci.2, Cii.2 e Ciii.2 }\end{array}$} & OWA_B \\
\hline & & & OWA_C \\
\hline \multirow[t]{2}{*}{3} & WLC & $\begin{array}{l}\text { Combinação de mapas do nível } 3 \text { para gerar os mapas B1, } \\
\text { C1, e C2, utilizando os pesos dos respectivos critérios }\end{array}$ & $\begin{array}{l}\text { WLC_B1 } \\
\text { WLC_C1 } \\
\text { WLC_C2 } \\
\end{array}$ \\
\hline & OWA & $\begin{array}{l}\text { Combinação dos mapas do Nível 3, gerando } 6 \text { cenários de } \\
\text { avaliação - C3i, C3ii, C3iii, C3iv, C3v, C3vi }\end{array}$ & OWA_C3 \\
\hline
\end{tabular}


para gerar o Mapa B1 que representa o critério de Índices Combinados de Defeitos. Em seguida foi realizada a combinação OWA_B (Tabela 2) do nível 2 para gerar os Mapas Bi, Bii e Biii, representando três cenários de diferentes riscos (Pontos de decisão na Figura 3). Para compor o Índice Combinado de Defeitos (Mapa B1) adotou-se o método proposto por Lee \& Park (1997) para priorização de pavimentos (Equação 4). É um método de combinação ponderada (WLC B1) que agrega a importância relativa de cada tipo de defeito aos valores normalizados de severidade e extensão. FCI (Weighted Fuzzy Condition Index) é Índice de Condição Ponderado Fuzzy, $w_{k i}$ é o peso subjetivo do defeito $\mathrm{k}$ com nível de severidade $\mathrm{i}, \mathrm{A}_{\mathrm{ki}}$ é a extensão do defeito $\mathrm{k}$ com severidade $\mathrm{i}$ e $\mathrm{S}_{\mathrm{i}}$ são valores subjetivos do nível de severidade i (Bandara \& Gunaratne, 2001).

$$
F C I=\frac{\sum_{i=1}^{3} \sum_{k=1}^{n} w_{k i} \cdot A_{k i} \cdot s_{i}}{\sum_{i=1}^{3} \sum_{k=1}^{n} w_{k i} \cdot A_{k i}}
$$

Os cenários $\mathrm{Bi}$, Bii e Biii, que representam o grupo B (Fatores Associados a Questões Técnicas e Operacionais) foram gerados através do procedimento OWA_B, combinando os scores ponderados dos fatores dos mapas B1 (Índice Combinado de Defeitos) e B2 (Índice da Condição dos Pavimentos) e contando com novos conjuntos de pesos (Order Weights), conforme a Tabela 3. As Figuras 5e, 5f, 5g, 5h e 5i apresentam, respectivamente, os mapas do grupo $\mathrm{B}$ do Nível 2, B1, B2 e os resultantes do Nível 1, Bi, Bii e Biii.

O cenário de risco neutro Bi (Figura $5 \mathrm{~g}$, com tradeoff total) apresentou valores bem variados dentro da escala considerada, pois os scores baixos são compensados por scores altos, o que resulta em valores intermediários. Para efeito de exemplo, uma determinada seção apresentou no mapa B1 (Figura 5e), cor amarelo (score $=0,571)$, no mapa B2 (Figura 5f) cor vermelha (score $=0,90)$ e no cenário Bi apresentou cor laranja (score $=0,654)$, indicando a neutralidade da avaliação que considera dois conjuntos de pesos, o primeiro dos

Tabela 3: Cenários de avaliação empregados para gerar mapas do Grupo B

\begin{tabular}{ccccc}
\hline Cenários & Order Weights & $\begin{array}{c}\text { AND- } \\
\text { Ness }\end{array}$ & $\begin{array}{c}\text { Trade- } \\
\text { off }\end{array}$ & $\begin{array}{c}\text { Atitudes de } \\
\text { Risco }\end{array}$ \\
\hline Bi & $(0,50 ; 0,50)$ & 0,50 & 1,00 & $\begin{array}{c}\text { Risco neutro } \\
\text { Trade-off total }\end{array}$ \\
\hline Bii & $(1,00 ; 0,00)$ & 1,00 & 0,00 & $\begin{array}{l}\text { Risco mínimo } \\
\text { Sem Trade-off }\end{array}$ \\
\hline Biii & $(0,00 ; 1,00)$ & 0,00 & 0,00 & $\begin{array}{l}\text { Risco máximo } \\
\text { Sem Trade-off }\end{array}$ \\
\hline
\end{tabular}

critérios e o segundo dos Order Weights com igual valor para todos os scores. O cenário de risco mínimo Bii (Figura 5h), por buscar os menores scores, possui uma grande porcentagem de cores verdes (scores entre $0,0$ e 0,2$)$. O cenário de risco máximo Biii (Figura 5i), ao contrário do mínimo, apresenta valores mais elevados. Se compararmos o cenário de risco mínimo Bii com o cenário de risco máximo Biii (ambos sem trade-off), no primeiro somente em $2 \%$ das seções há valores maiores que 0,4 , enquanto no segundo aumentou para $35 \%$, sendo a distribuição dessas seções muito mais ampla no cenário Biii_n.

\subsection{Combinações de critérios do Grupo C (Níveis 3 e 2) e Grupo D}

$\mathrm{Na}$ combinação dos critérios do grupo $\mathrm{C}$ realizaram-se os seguintes processos:

- WLC_C1: agrupamento dos scores normalizados dos critérios $\mathrm{C} 1.1$ a C1.3, dando origem ao mapa $\mathrm{C} 1$ do Nível 2 (Figura 6a);

- WLC_C2: agrupamento dos scores normalizados dos critérios $\mathrm{C} 2.1$ a C2.7, dando origem ao mapa C2 do Nível 2 (Figura 6b);

- OWA_C3: agrupamento dos scores ponderados dos critérios C3.1 a C3.5, contando com novos conjuntos de pesos (Order Weights - Tabela 4), originando os cenários $\mathrm{C} 3 \mathrm{i}, \mathrm{C} 3 \mathrm{ii}, \mathrm{C} 3 \mathrm{iii}, \mathrm{C} 3 \mathrm{iv}$, C3v e C3vi do Nível 2;

- OWA_C: agrupamento dos mapas C1, C2 e C3i (risco neutro), contando com novos conjuntos de pesos (Order Weights - Tabela 5). A combinação deu origem aos cenários $\mathrm{Ci}$, Cii e Ciii do Nível 1, representando o Grupo C, Fatores Associados à Localização.

Além dos grupos A, B e C, o grupo D também faz parte do nível 1 , porém não contém outros níveis mais baixos da estrutura hierárquica, sendo o mapa $\mathrm{D}$ (Figura 61) desenvolvido diretamente através de scores normalizados referentes aos custos de M\&R de pavimentos. Portanto, a Figura 6 apresenta os mapas do grupo C, no nível 2: C1, C2; C3i, C3ii, C3iii, C3iv, C3v, C3vi e os resultantes do Nível 1: Ci, Cii e Ciii, e o mapa do grupo D (Nível 1). Portanto, a Figura 6 apresenta os mapas do grupo $\mathrm{C}$, no nível 2: $\mathrm{C} 1, \mathrm{C} 2$; C3i, C3ii, C3iii, C3iv, C3v, C3vi e os resultantes do Nível 1: Ci, Cii e Ciii, e o mapa do grupo D (Nível 1). 


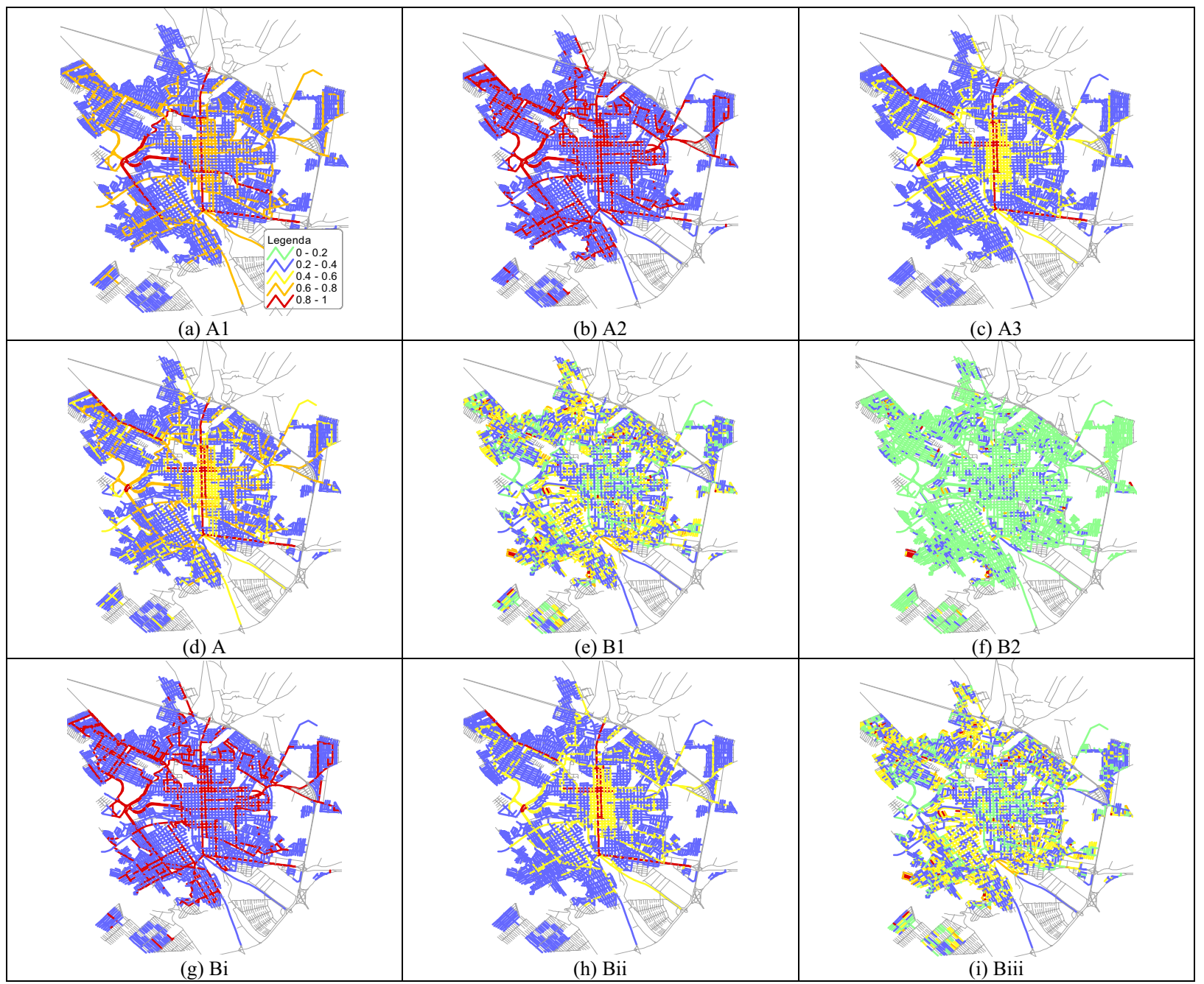

Figura 5. Mapas dos Grupos A e B

Tabela 4. Cenários de avaliação empregados para gerar os mapas do critério C3

\begin{tabular}{ccccc}
\hline Cenários & Order Weights & ANDNess & Trade-off & Pontos de decisão \\
\hline C3i & $(0,20 ; 0,20 ; 0,20 ; 0,20 ; 0,20)$ & 0,50 & 1,00 & i: risco neutro - trade-off total \\
\hline C3ii & $(1,00 ; 0,00 ; 0,00 ; 0,00 ; 0,00)$ & 1,00 & 0,00 & ii: risco mínimo- sem trade-off \\
\hline C3iii & $(0,00 ; 0,00 ; 0,00 ; 0,00 ; 1,00)$ & 0,00 & 0,00 & iii: risco máximo - sem trade-off \\
\hline C3iv & $(0,50 ; 0,20 ; 0,15 ; 0,10 ; 0,05)$ & 0,75 & 0,60 & iv: baixo risco - trade-off parcial \\
\hline C3v & $(0,05 ; 0,10 ; 0,15 ; 0,20 ; 0,50)$ & 0,25 & 0,60 & v: alto risco - trade-off parcial \\
\hline C3vi & $(0,30 ; 0,15 ; 0,10 ; 0,15 ; 0,30)$ & 0,50 & 0,79 & vi: risco neutro-trade-off parcial \\
\hline
\end{tabular}

Tabela 5: Cenários de avaliação empregados para gerar os mapas do Grupo C

\begin{tabular}{|c|c|c|c|c|}
\hline Procedimento & Cenário & Order Weights & \multicolumn{2}{|c|}{ Cenários combinados } \\
\hline \multirow{3}{*}{ OWA_C } & $\mathrm{Ci}$ & $(0,33 ; 0,33 ; 0,33)$ & \multirow{3}{*}{$\begin{array}{l}\mathrm{C} 1 \\
\mathrm{C} 2 \\
\mathrm{C} 3 \mathrm{i}\end{array}$} & \multirow{3}{*}{$\begin{array}{l}\text { WLC } \\
\text { WLC } \\
\text { WLC }\end{array}$} \\
\hline & Cii & $(1,00 ; 0,00 ; 0,00)$ & & \\
\hline & Ciii & $(0,00 ; 0,00 ; 1,00)$ & & \\
\hline
\end{tabular}

Os cenários de risco mínimo (ii) ou máximo (iii) limitam, inferior ou superiormente, a tomada de decisão, enquanto que no cenário de risco neutro (i) os scores são compensados, resultando em valores intermediários. No mapa Ci (Figura $6 \mathrm{i}$ - risco neutro) predominam as tonalidades verdes e azuis, com algumas seções em amarelo na região central e em locais pró- ximos a postos de saúde (valores baixos, com apenas $4,5 \%$ de valores nulos). Também é perceptível a presença de maior prioridade (cor laranja) em seções próximas a rodovias. O mapa Cii (Figura $6 \mathrm{j}$ - risco mínimo) é quase totalmente verde com aproximadamente $98 \%$ das seções com scores iguais a zero, salientando algumas seções próximas ao terminal rodovi- 


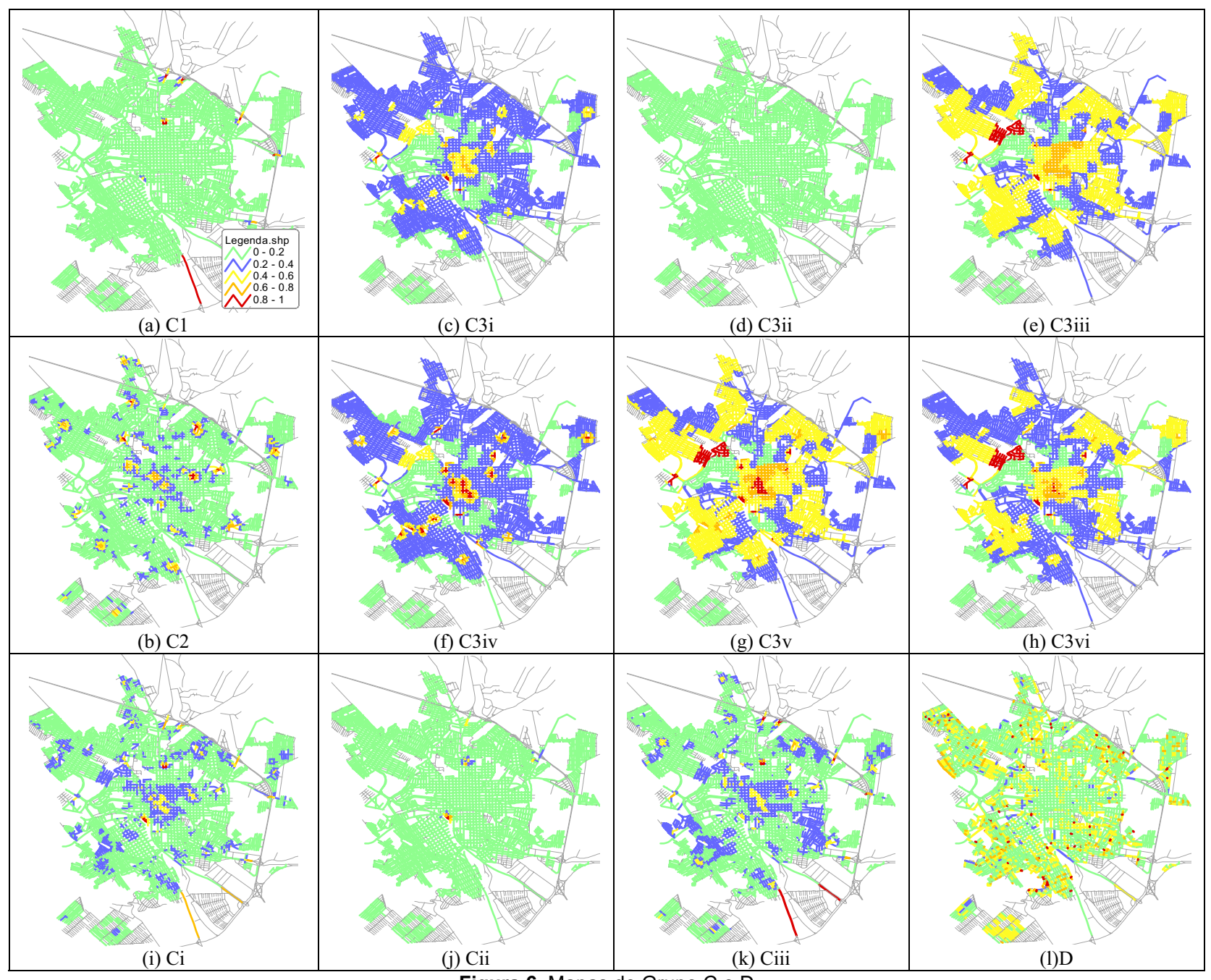

Figura 6. Mapas do Grupo C e D

Tabela 6. Estatísticas dos mapas do nível 1

\begin{tabular}{ccccc}
\hline Mapa & Média & Desvio Padrão & \% de valores zero & Tipo de Avaliação \\
\hline $\mathrm{A}$ & 0,621 & 0,188 & 0 & Risco Neutro \\
\hline Bi_n & 0,291 & 0,132 & 1 & Risco Neutro \\
\hline Bii_n & 0,074 & 0,124 & 37 & Risco Mínimo \\
\hline Biii_n & 0,355 & 0,146 & 1 & Risco Máximo \\
\hline Ci & 0,164 & 0,099 & 4,6 & Risco Neutro \\
\hline Cii & 0,006 & 0,052 & 98 & Risco Mínimo \\
\hline Ciii & 0,188 & 0,102 & 5 & Risco Máximo \\
\hline D & 0,147 & 0,063 & 73 & $*$ \\
\hline
\end{tabular}

* Scores normalizados de Custo de M\&R.

ário e rodovias. O mapa Ciii (risco máximo) difere em relação ao resultado final, com scores mais elevados e somente 5\% das seções com scores nulos (sem prioridade de intervenção). O mapa D (Figura 61) apresenta $73 \%$ de seções com valores nulos, onde as atividades previstas são de não fazer nada, levando, portanto, o custo a zero e $25 \%$ das seções possuem scores maior que 0,4 , indicando a prioridade de intervenção. As estatísticas dos valores gerados em cada mapa do nível 1 são apresentadas na Tabela 6.

\subsection{Mapas finais de prioridades}

As combinações dos fatores associados à hierarquia viária (grupo $\mathrm{A}$ ), fatores associados às questões técnicas e operacionais (grupo B), fatores associados à localização (grupo C) e fatores associados aos custos (grupo D) originam os mapas finais, ou seja, os mapas de ranking das seções de vias pavimentadas, de acordo com a ordem crescente dos scores e de prioridades. Através do procedimento OWA_G (Tabela 2), são desenvolvidos seis cenários de avaliação em duas etapas. Enquanto que a primeira etapa combina o mapa A, os cenários $\mathrm{Bi}$ e $\mathrm{Ci}$ e o mapa $\mathrm{D}$, todos de risco neutro, a segunda combina dois cenários neutros $\mathrm{A}$ e $\mathrm{D}$, um cenário de risco mínimo Bii e um cenário de risco má- 
ximo Ciii. Aplicando novos conjuntos de pesos ( $\mathrm{Or}-$ der Weights - Tabela 7) em ordem crescente dos scores ponderados correspondentes aos grupos de fatores A, B, C e D, são desenvolvidos os mapas Pi.1, Pii.1 e Piii.1 na primeira etapa e Pi.2, Pii.2 e Piii.2 na segunda etapa. A Tabela 7 mostra os seis cenários utilizados e a Figura 7 apresenta os mapas finais de avaliação.

$\mathrm{Na}$ primeira etapa, foi realizada, em todos os níveis, uma análise de decisão neutra. Entretanto, a segunda etapa combina um cenário A neutro, resultante de procedimento WLC, um cenário B pessimista (risco mínimo), um cenário $\mathrm{C}$ otimista (risco máximo) e um cenário $\mathrm{D}$, gerado através de scores normalizados. Optou-se por uma atitude conservadora, adversa ao risco em relação às questões técnicas e operacionais (cená- rio B, menores scores são valorizados), no qual é garantido que todas as seções de vias, com scores altos, sejam identificadas. Ainda, optou-se por uma atitude neutra em relação à hierarquia viária (cenário A) e aos custos de M\&R (cenário D) e uma decisão otimista, em que se valorizam os scores mais elevados quanto à localização das seções de pavimentos (cenário C). Esta decisão favorece a identificação das vias com problemas técnicos e operacionais mais graves, ou seja, defeitos e condição do pavimento que prejudicam o tráfego na via.

A hierarquia viária e os custos da estratégia de M\&R são considerados de forma neutra e, ainda, valoriza pelo menos um aspecto de localização que esteja colaborando para a priorização da via. As estatísticas

Tabela 7. Cenários de avaliação empregados para gerar os mapas finais de priorização

\begin{tabular}{|c|c|c|c|}
\hline Procedimento & Cenário & $\begin{array}{c}\text { Order } \\
\text { Weights }\end{array}$ & $\begin{array}{c}\text { Cenários } \\
\text { combinados }\end{array}$ \\
\hline \multirow{6}{*}{ OWA_G } & Pi.1 & $(0,25 ; 0,25 ; 0,25 ; 0.25)$ & \multirow{3}{*}{$\begin{array}{ll}\text { A: } & \text { WLC; } \\
\text { Bi: } & \text { WLC; } \\
\text { Ci: } & \text { WLC; } \\
\text { D: } & \text { Scores normalizados. }\end{array}$} \\
\hline & Pii.1 & $(1,00 ; 0,00 ; 0,00 ; 0,00)$ & \\
\hline & Piii.1 & $(0,00 ; 0,00 ; 0,00 ; 1,00)$ & \\
\hline & Pi.2 & $(0,25 ; 0,25 ; 0,25 ; 0.25)$ & \multirow{3}{*}{$\begin{array}{l}\text { WLC; } \\
\text { Risco mín; } \\
\text { Risco máx; } \\
\text { Scores normalizados. }\end{array}$} \\
\hline & Pii.2 & $(1,00 ; 0,00 ; 0,00 ; 0,00)$ & \\
\hline & Piii.2 & $(0,00 ; 0,00 ; 0.00 ; 1,00)$ & \\
\hline
\end{tabular}

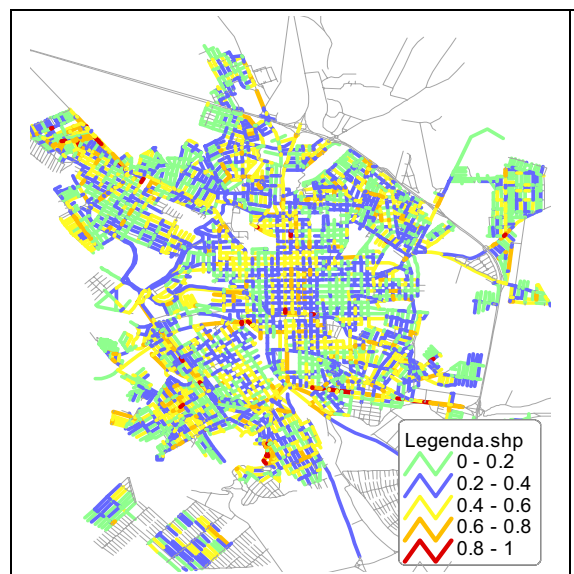

(a) Pi.1

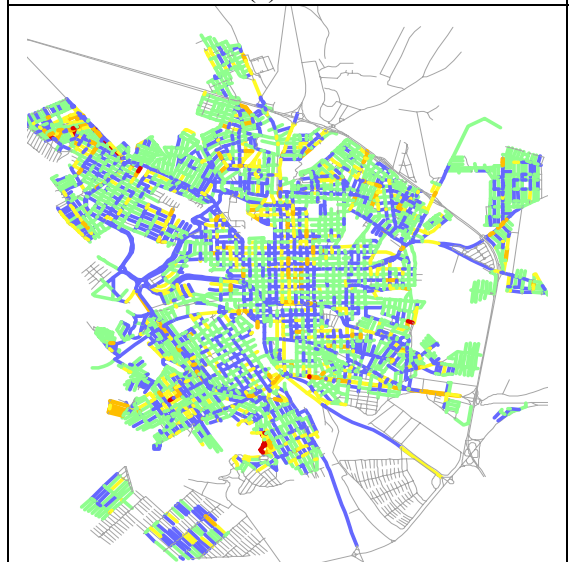

(d) Pi.2

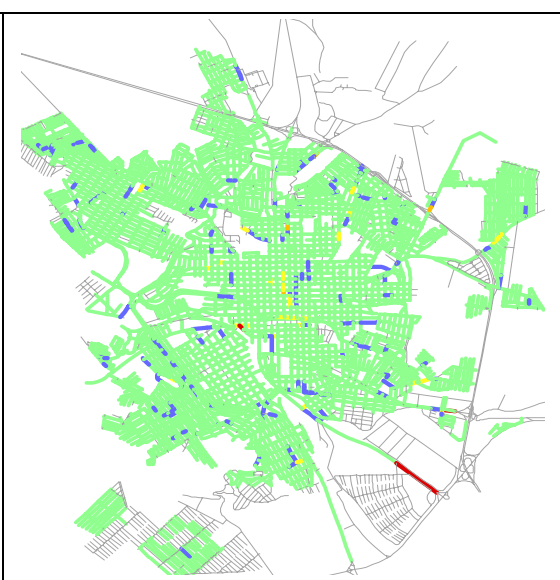

(b) Pii.1

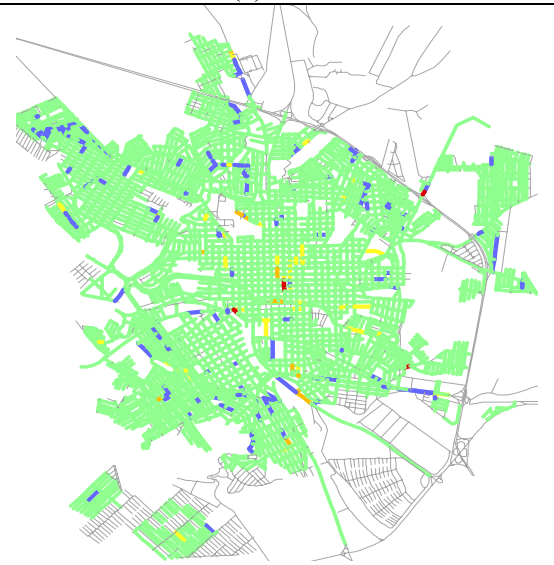

(e) Pii.2

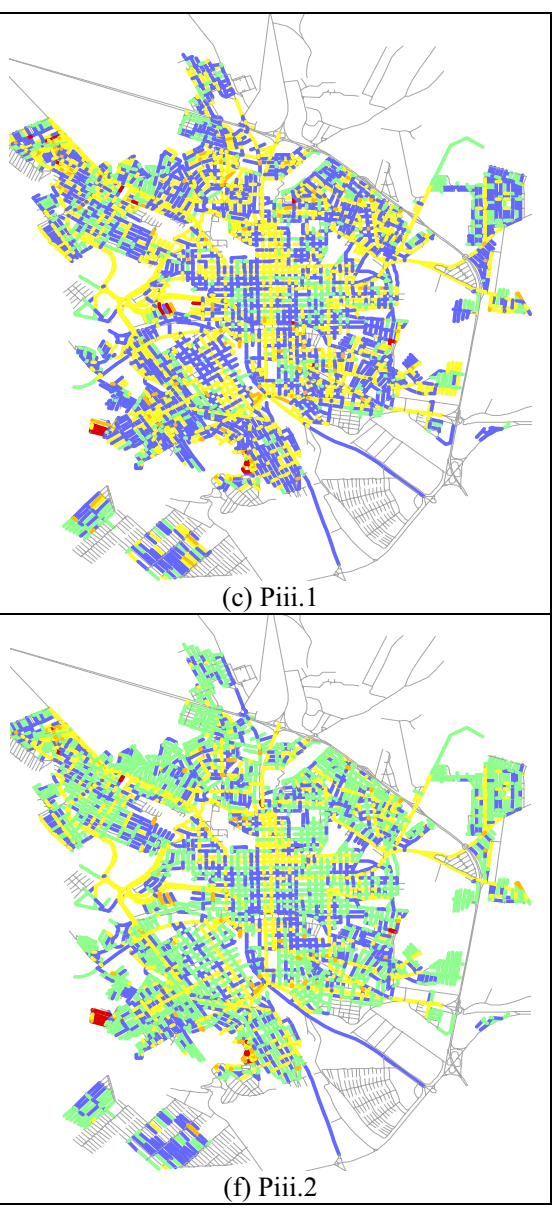

(f) Piii.2

Figura 7. Mapas finais de priorização de vias pavimentadas 
dos valores gerados em cada mapa final são apresentadas na Tabela 8 .

Tabela 8. Estatísticas dos mapas finais de priorização

\begin{tabular}{ccccc}
\hline Mapa & Média & $\begin{array}{c}\text { Desvio } \\
\text { Padrão }\end{array}$ & $\begin{array}{c}\text { \% de } \\
\text { valores } \\
\text { zero }\end{array}$ & $\begin{array}{c}\text { Tipo de } \\
\text { Avaliação }\end{array}$ \\
\hline Pi.1 & 0,322 & 0,168 & 0 & Risco Neutro \\
\hline Pii.1 & 0,018 & 0,073 & 92 & Risco Mínimo \\
\hline Piii.1 & 0,349 & 0,137 & 0 & Risco Máximo \\
\hline Pi.2 & 0,221 & 0,181 & 1 & Risco Neutro \\
\hline Pii.2 & 0,020 & 0,086 & 93 & Risco Mínimo \\
\hline Piii.2 & 0,247 & 0,182 & 1 & Risco Máximo \\
\hline
\end{tabular}

Conforme especificado na Tabela 8 , os cenários Pi.1 e Pi.2 são resultados de análises de risco neutro, Pii.1 e Pii.2 de risco mínimo e Piii.1 e Piii.2 de risco máximo. De modo geral, observa-se que os mapas gerados na primeira etapa, em que foram considerados somente cenários de risco neutro, possuem valores mais elevados em relação aos mapas gerados na segunda etapa. Observando a Figura 7 e comparando os cenários de risco neutro Pi.1 e Pi.2, o primeiro não possui valores iguais a zero enquanto que o segundo já tem $1 \%$ de valores nulos.

Em relação à escolha de seções de pavimentos, os cenários Pii.1 e Pii.2, de risco mínimo, oferecem poucas seções a serem priorizadas, sendo que aproximadamente $93 \%$ das seções apresentam scores nulos em ambos os mapas (seções verdes). Os mapas de risco máximo, Piii.1 e Piii.2, já mostram uma grande quantidade de seções amarelas (36\% no Piii. 1 e $35 \%$ no Piii.2) e laranja ( $3 \%$ no Piii. 1 e $2 \%$ no Piii.2) e uma pequena parcela de seções vermelhas, que não existiam nos mapas anteriores. A seleção das seções, neste caso, torna-se mais abrangente, pois são maiores as opções de priorização. Em termos práticos, maiores investimentos seriam realizados com os cenários de risco máximo.

\section{CONCLUSÕES}

O objetivo geral deste trabalho foi apresentar um modelo de priorização de vias, integrando as técnicas de Análise Multicritério com as ferramentas de um Sistema de Informação Geográfica para apoiar as decisões envolvidas na conservação de pavimentos viários. O modelo mostrou-se eficiente em várias etapas do processo, destacando-se a flexibilidade devido à possibilidade de modificar a estrutura hierárquica desenvolvida, no instante que se pretende retirar ou adicionar algum critério de decisão. A aplicação do AHP possibilitou a averiguação do grau de consistência dos julgamentos, comprovando a eficiência da avaliação e a relevância dos critérios considerados, uma análise considerada de extrema importância quando se trata de tomada de decisão com múltiplos critérios. Entre- tanto, a importância atribuída a cada um dos critérios pode ser reconsiderada, dependendo das necessidades reais de cada cidade em que o modelo seja aplicado.

A geração de alternativas de priorização de vias pavimentadas depende de forma significativa da opinião do decisor (do especialista ou técnico responsável pelo planejamento dos serviços), principalmente quando são elaborados os diversos cenários de avaliação e faz-se a opção por um deles. Por outro lado, não se pode afirmar qual dos cenários gerados, através da variação de trade-off em várias etapas, é a melhor alternativa, pois a decisão final deve considerar as necessidades de cada órgão administrativo e a disponibilidade de recursos para a execução dos serviços selecionados. Se existe disponibilidade maior de recurso para ser investido, então, pode-se considerar uma análise de risco máximo satisfatória, pois gera mapas com número mais elevado de scores altos (entre 0,6 e 1,0) do que um cenário de risco mínimo, tornando a priorização mais abrangente.

Do ponto de vista instrumental o modelo apresentado mostra-se interessante pelo fato de considerar de forma simples os conceitos de priorização de vias e ainda integrá-lo numa ferramenta poderosa de análise espacial. A exploração do modelo pode assumir formato de utilização prática, tal como auxiliar administradores de órgãos governamentais na função de avaliar e planejar as intervenções de conservação em vias urbanas pavimentadas. Pelo estudo de caso apresentado a aplicabilidade do modelo ficou demonstrada, quer na perspectiva da sua operacionalidade quer na perspectiva da sua utilidade.

\section{AGRADECIMENTOS}

Os autores agradecem a CAPES (Coordenação de Aperfeiçoamento de Pessoal de Nível Superior) pelo apoio financeiro que subsidiou o desenvolvimento desse trabalho.

\section{REFERÊNCIAS BIBLIOGRÁFICAS}

Albuquerque, F. S. (2007). Sistema de gerência de pavimento para departamentos de estradas do nordeste brasileiro. Tese (Doutorado). Universidade Federal do Rio Grande do Sul. Porto Alegre, RS.

Bana e Costa, C. A.; Vansnick, J. C. (2007). A critical analysis of the eigenvalue method used to derive priorities in AHP. European Journal of Operational Research vol. 187, no. 3, pp. 1422-1428.

Bana e Costa, C. A.; Vansnick, J. C. (1994). MACBETH - An Interactive Path Towards the Construction of Cardinal Value Functions, International Transactions in Operational Research, vol. 1, n. 4, p. 489-500.

Bandara, N.; Gunaratne, M. (2001). Current and Future Pavement Maintenance Prioritization Based on Rapid Visual Condition Evaluation. Journal of Transportation Engineering. March/April, pp.116-123.

Cafiso, S.; Graziano, A.; Kerali, H. R.; Odoki, J. B. (2002). Multicriteria Analysis Method for Pavement Maintenance Management. Transportation Research Record 1816. TRB. Washington, D.C., pp7384.

Chandran, S.; Isaac, K. P; Veeraragavan, A. (2007). Prioritization of LowVolume Pavement Sections for Maintenance by Using Fuzzy Logic. Journal of the Transportation Research Board. Transportation Research Record.

Chen, X.; Weissmann, J.; Dossey, T.; Hudson, W. R. (1993). URMS: graphical urban roadway management system at network level. 
Transportation Research Record 1397. TRB. Washington, D.C., pp103-111.

Costa, M.S.; Silva, A.N.R.; Ramos, R.A.R. (2004). Indicadores de mobilidade urbana sustentável para o Brasil e Portugal. In: Contribuições para o Desenvolvimento Sustentável em Cidades Portuguesas e Brasileiras. Ed.Coimbra, Portugal : Almedina. V.1, p. 83-97.

Eastman, J. R.; Jiang, H.; Toledano, J. (1998). Multi-criteria and multiobjetive decision making for land allocation using GIS. In: Beinat, E.; Nijkamp, P. (Eds), Multicriteria Analysis for Land-Use Management. Dordrecht: Kluwer Academic Publishers. P. 227-251.

Easton, A. (1973). Complex Managerial Decision Involving Multiple Objectives. John Wiley \& Sons, New York, NY, USA

Jankowski, P.; Andrienko, N.; Andrienko, G. (2001). Map-centred exploratory approach to multiple criteria spatial decision making. International Journal of Geographical Information Science, v.15, n.2, p.101-127, Mar.

Lee, D. H.; Park, D. (1997). An efficient algorithm for fuzzy weighted average. Fuzzy Sets and Systems. P. 39-45.

Lima, J. P. (2007). Modelo de decisão para a priorização de vias candidatas às atividades de manutenção e reabilitação de pavimentos. 167 p. Tese (Doutorado). Escola de Engenharia de São Carlos, Universidade de São Paulo, São Carlos.

Lima, J.P.; Lopes, S.B.; Zanchetta, F.; Anelli, R.L.S.; Fernandes Jr, J.L. (2004). O Uso de SIG em gerência de Infra-estrutura Urbana de Transportes: Estudo de Caso em São Carlos - SP. In: Contribuições para o Desenvolvimento Sustentável em Cidades Portuguesas e Brasileiras. Ed.Coimbra, Portugal: Almedina V.1, p. 146159.

Malczewski (1999). GIS and multicriteria decision analysis. New York: John Wiley \& Sons, Inc.

Mello, J. C. C. B. S.; Gomes, E. G. ; Lins, M. P. E.; Vieira, L. A. M. (2001) Um caso de estudo de integração SIG-DEA-MCDA: a influência de uma instituição de ensino superior em vários municípios do estado do rio de janeiro. Revista Científica Investigação Operacional. Vol nº 21 .

Misra, A.; Roohanirad, A.; Somboonyanon, P. (2003). Guidelines for a Roadway Management System (RMS) for Local Governments. Final Report. MTC - Midwest Transportation Consortium. (Disponível em: $<$ http://www.ctre.iastate.edu/mtc/reports/RMS.htm> Acesso em: 25 out 2005).

Mohammad, K.; Herring, A.; Chee, K. M. (2004). An Evaluation of Various Prioritization Methods for Effective Pavement Management: A Canadian Airport Case Study. Worldwide Airport Technology Transfer Conference. Atlantic City, New Jersey, USA.

Osgood, C.E.; Suci, G.J.; Tannenbaum, P.H. (1957). The Measurement of Meaning. University of Illinois Press, Urbana, IL, USA

Pereira, P.; Miranda, V. (1999). Gestão da conservação dos pavimentos rodoviários. Braga, Portugal. Psychology, vol. 15, p. 234-281

Ramos, R. A. R. (2000). Localização industrial-um modelo espacial para o noroeste de Portugal. Tese (Doutorado). Universidade do Minho. Braga, Portugal.

Rodrigues, D.S.; Mendes, J.F.G.; Lima, J.P.; Ramos, R.A.R. (2004). Uma abordagem multicritério para a avaliação da acessibilidade. In: Contribuições para o Desenvolvimento Sustentável em Cidades Portuguesas e Brasileiras. Almedina - Coimbra, Portugal. V.1, p. 98-117.

Saaty, T. (1980). The Analytic Hierarchy Process. New York. McGraw Hill.

Saaty, T. L. (1977), "A Scaling Method for Priorities in Hierarchical Structures", Journal of Mathematical.

Salomon, V.P.; Montevechi, J. A. B.; Pamplona, E. O. (1999). Justificativas para aplicação do método de análise hierárquica. In: XIX Encontro Nacional de Engenharia de Produção -ENEGEP, Rio de Janeiro.

Stillwell, W.G.; Seaver, D.A.; Edwards, W. (1981). A Comparison of Weight Approximation Techniques in Multiattribute Utility Decision Making. Organization Behavior and Human Performance, 28(1), 62-77.

Visconti, T. S. (2000). O Sistema Gerencial de Pavimentos do DNER. Departamento Nacional de Estradas de Rodagem. DNER. (Disponível em: http://www1.dnit.gov.br/arquivos_internet/ipr/ipr_new/manuais/ pms 99.pdf. Acesso em: 20 set 2006)

Voogd, H. (1983). Multicriteria evaluation for urban and regional planning. London: Pion Ltd.

Yager, R. R. (1988). On ordered weighted averaging aggregation operators in multicritério decision making. IEEE Transactions on Systems, Man and Cybernetics, v. 8, n. 1, p. 183-190.
Zadeh, L. A. (1965). Fuzzy Sets. Information and Control, Vol.8. pp.338353.

Zambon, K.L. (2004). Localização de usinas termoelétricas utilizando sistema de informação geográfica e métodos de decisão multicritério. Tese (doutorado). Dissertação (Mestrado). Universidade de São Paulo, Escola de Engenharia de São Carlos, Departamento de Transportes. São Carlos, SP

Zietsman, J.; Rilett, L.R.; Kim S. (2003). Sustainable transportation performance measures for developing communities. Report 167128 Supported by general revenues from the State of Texas. Texas Transportation Institute. 